Proc. of the 15th Int. Workshop on Slow Positron Beam Techniques and Applications, Prague, September 2-6, 2019

\title{
PALS Avalanche - A New PAL Spectra Analysis Software
}

\author{
K. DUlski*, On Behalf of The J-PET COLlaboration \\ Jagiellonian University, Faculty of Physics, Astronomy and Applied Computer Science,
} S. Lojasiewicza 11, 30-348 Krakow, Poland

\begin{abstract}
A novel concept for tomography of the human body developed by the Jagiellonian Positronium Emission Tomography (J-PET) project provides the possibility to combine metabolic information collected by standard PET with structural information obtained from positronium lifetime. This results in a morphometric image. To this end, there is a need to develop software compatible with the J-PET Framework for fast online analysis during imaging. PALS Avalanche is a software developed on UNIX system and based on ROOT software, which allows one to decompose positronium annihilation lifetime spectra in the form of a set of single time differences and histogram. Performance of the PALS Avalanche will be tested by analysing simulated PAL spectra.
\end{abstract}

DOI: $10.12693 /$ APhysPolA.137.167

PACS/topics: 29.90.+r 02.70.-c 02.60.-x

\section{Introduction}

The J-PET project [1-5] was developed with intention to construct a Positron Emission Tomograph (PET) based on plastic scintillators that will be able to compete with commercial PET detectors in terms of quality of created image. However, special construction of J-PET allows one to incorporate other applications, based on positron emission phenomenon.

One of the possible applications of the J-PET detector is to use it also as a positron annihilation lifetime spectrometer (PALS). Combining two techniques like PET and PALS, brings the possibility to create a new class of imaging, contained in a concept of morpohometric image $[6,7]$. For real-time imaging, comparable with commercial solutions, PET detectors require one to use highspeed technology and dedicated software. Therefore, for fast analysis, monitoring and imaging of data registered with the J-PET detector specific software was designed, the so called J-PET Framework [8]. This software, developed in $\mathrm{C}++$ and based on BOOST and ROOT libraries $[9,10]$, allows one to reconstruct events registered in the J-PET detector and classify them in appropriate channel of decay. Therefore, thanks to Framework structure, it is possible to find events containing positronium annihilation and further on to create PAL spectrum simultaneously with tomographic image of the measured setup.

In order to create morphometric image, consisting of metabolic map from PET and lifetime map from PALS, one needs a measure of lifetime, that can be a value of response for each voxel in the lifetime map [6]. To obtain a real-time metabolic image one needs a compatible software with the J-PET framework that will be able to analyse lifetime spectra simultaneously with the data

\footnotetext{
* corresponding author; e-mail:
}

kamil.dulski@doctoral.uj.edu.pl collection. This need was met with the PALS Avalanche software, that has been compared with widely used LT software before [11-13].

\subsection{Details of the fitting procedure}

Mathematical model that is used for the analysis is the sum of exponential distributions convoluted with a sum of Gaussian distributions [14]. It can be described as $F(t)=y_{0}$

$$
\text { +Area } \sum_{i=0}^{N} \sum_{j=0}^{M} P_{i} R_{j}\left(\exp \left(\tau_{i}\right) * \operatorname{Gauss}\left(t_{0}^{(j)}, \sigma_{j}\right)\right)(t),
$$

where

$$
\begin{aligned}
& \left(\exp \left(\tau_{i}\right) * \operatorname{Gauss}\left(t_{0}^{(j)}, \sigma_{j}\right)\right)(t)=\frac{1}{2 \tau} \exp \left(\frac{\sigma^{2}}{2 \tau^{2}}-\frac{t-t_{0}}{\tau}\right) \\
& \quad \times\left(\operatorname{erf}\left(\frac{t-t_{0}-\frac{\sigma^{2}}{\tau}}{\sqrt{2} \sigma}\right)-\operatorname{erf}\left(\frac{-t_{0}-\frac{\sigma^{2}}{\tau}}{\sqrt{2} \sigma}\right)\right),
\end{aligned}
$$

$y_{0}$ is the background, Area is the normalization factor, $N$ and $M$ are the numbers of lifetime and resolution components respectively, $P_{i}$ and $R_{j}$ are intensities of lifetime and resolution component respectively with restriction that $\sum_{i} P_{i}=1, \sum_{j} R_{j}=1$. The parameter $t_{0}$ is the mean of the Gaussian distribution with standard deviation $\sigma_{j}$ and $\tau_{i}$ is the mean lifetime of an exponential distribution.

The fitting procedure is based on the Minuit algorithm [15] which uses quasi-Newton method called the Davidon-Fletcher-Powell (DFP) to minimize cost function.

PALS Avalanche uses iterative procedure to help stabilize results from fitting, described fully in [13].

\section{Performance analysis}

In general, analysis of lifetime spectra using the PALS technique focuses on decomposition of the measured distribution of positron lifetimes. It is in fact a composition 
of resolution function which depends on the experimental setup used in the measurement, and a function of mean lifetimes of positron in the measured sample. Decomposition of measured PAL spectrum, is always connected with the so-called inverse problem, which creates ambiguity in obtained mean lifetime distribution.

For testing the performance of developed software for analysis of positron lifetime spectra it is useful, for proper interpretation of obtained results, to estimate uncertainty, introduced by the software itself, when decomposing measured spectrum.

\subsection{Simulation description}

To test the PALS Avalanche performance, simulations of PALS spectra were done. As an estimate of resolution function of experimental setup, single Gaussian distribution was assumed with mean equal to 6 ns, and standard deviation equal to $0.1 \mathrm{~ns}$. The distribution of positronium lifetime was described by the exponential function. Three positron components with different mean lifetime were simulated:

- First with mean lifetime equal to $0.125 \mathrm{~ns}$ and intensity of $15 \%$;

- Second with mean lifetime equal to $0.5 \mathrm{~ns}$ and intensity of $50 \%$;

- Third with mean lifetime equal to 5 ns and intensity of $35 \%$.

Pseudo-random generator from cstdlib library was used, for generating random numbers in the range of $[0,1]$. Exponential distribution was obtained using inverse cumulative distribution function of the exponential decay. Gaussian distribution was obtained using the Box-Muller transformation, which smeared previously obtained exponential distribution. Background was simulated as a uniform distribution in the range of $[-6,100] \mathrm{ns}$ and added to mean lifetime distribution smeared with resolution function. The simulated lifetime spectra were further fitted by the PALS Avalanche software. For statistical analysis, each distribution with given number of total counts, was simulated ten times.

\subsection{Measure of the uncertainty}

In analysis of PAL spectra there are many factors that can influence the results of analysis. Here dependence of few will be considered, like statistics, bin width of the fitted lifetime spectrum, fitting range, and at the end the difference between the initial parameters and the simulated ones.

As a measure of the systematic error introduced by PALS Avalanche, a weighted mean over estimated parameters was calculated for each component which then can be compared with the values assumed in the simulations generator as described in Sect. 2.1. These parameters include:
- Standard deviation and mean of assumed resolution function;

- Mean lifetime and intensity of assumed lifetime components,

where parameter $p$ can be any of the element from $\left\{\sigma, t_{0}, \tau_{1}, \tau_{2}, \tau_{3}, P_{1}, P_{2}, P_{3}\right\}$.

For each total number of counts set of ten distributions was simulated, so as a value of parameter $p$ compared with simulated $p^{\text {sim }}$, mean parameter was calculated as $p^{(i)}=\Sigma_{j=1}^{10} p_{j} / 10$. Weighted mean then was defined as:

$$
\text { weighted mean error }=\frac{\Sigma_{i}\left|p^{(i)}-p^{\text {sim }}\right|^{2}}{\Sigma_{i}\left|p^{(i)}-p^{\text {sim }}\right|},
$$

where weight is the $\left|p^{(i)}-p^{\text {sim }}\right|$ to decrease an impact of results that by accident are very close to the simulated value, where other parameters are not. Weighted mean error is therefore a measure of some maximal systematic error done by the software.

\subsection{Analysis results}

Having simulated distribution and a measure of uncertainty one estimate mean error introduced by analysis procedure itself and an impact of different factors. Example of simulated distribution with a fitted lifetime curve is shown in Fig. 1.
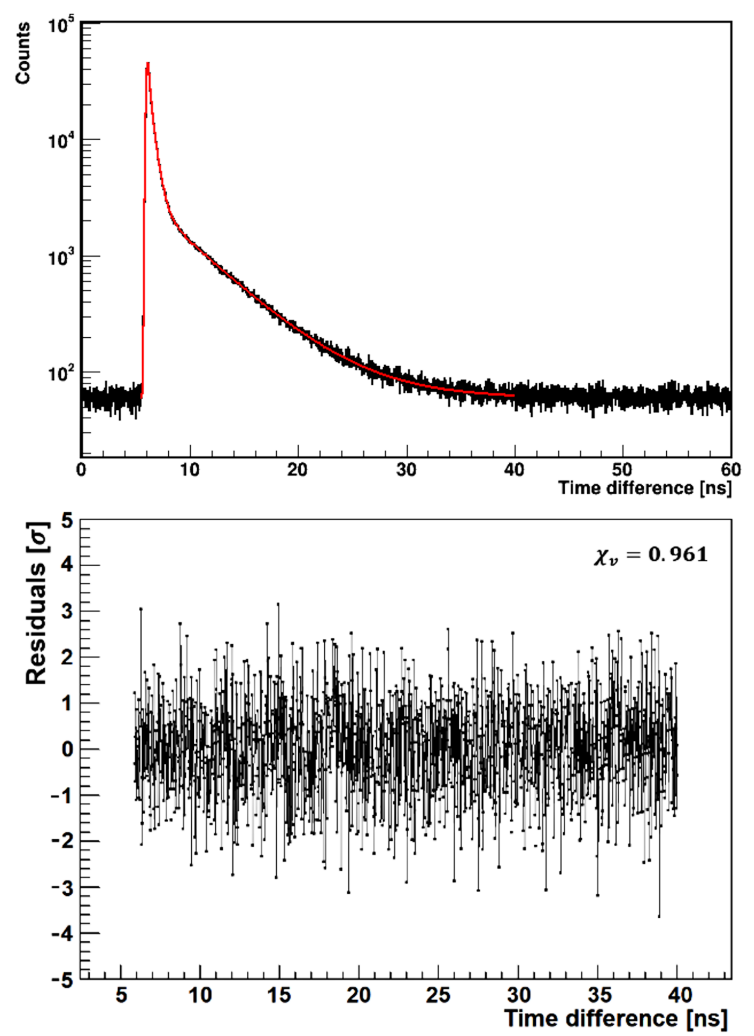

Fig. 1. Example of a simulated distribution obtained by generating $2 \times 10^{6}$ events with bin width equal to $0.02 \mathrm{~ns}$ with plot of residuals below. 


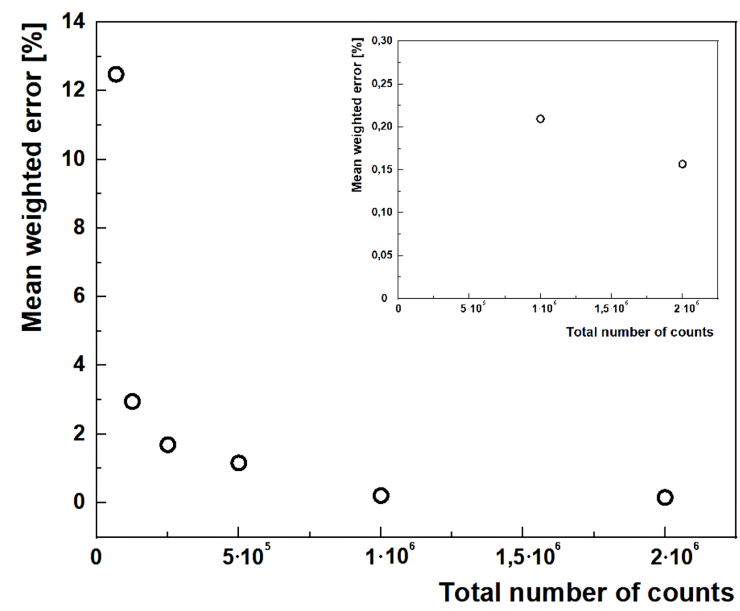

Fig. 2. Influence of statistic in spectrum on error made by fitting procedure.

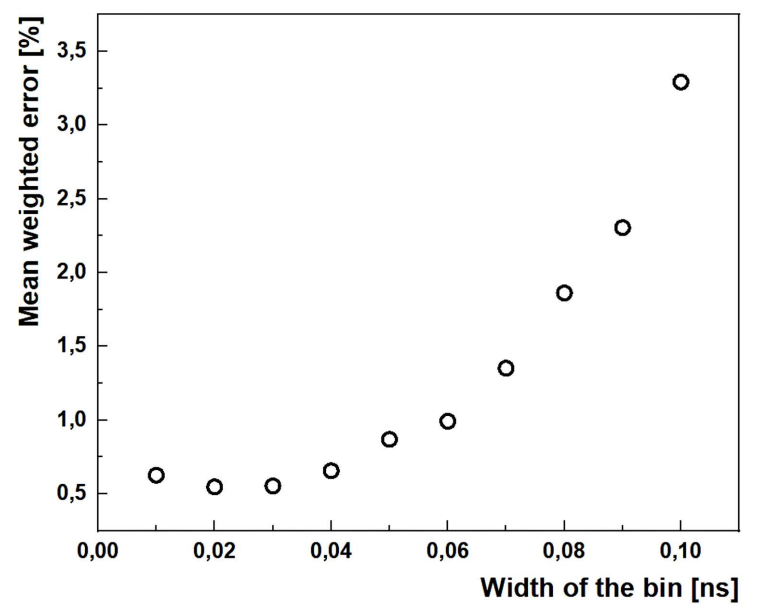

Fig. 3. Influence of the bin width of the lifetime spectrum on estimated parameters uncertainties.

To test the influence of statistics on the fitted parameters we fixed all the other factors to decrease their impact. The bin width of the lifetime spectra was assumed to be $0.02 \mathrm{~ns}$, fit was performed up to time difference equal to $40 \mathrm{~ns}$ and the initial parameters of the fit were equal to the simulated ones. Results are shown in Fig. 2.

As one can see it is enough to register total number of counts in order of 1 million for the uncertainty to be below $1 \%$. On the other hand, very low statistic below 100,000 , can lead to uncertainty greater than $10 \%$ in case of every fitted parameter. For the check of bin width influence the number of counts of the lifetime spectrum was fixed to $2 \times 10^{6}$ when all other factors were set as in the case of the previous check. Results of this analysis is shown in Fig. 3.

In Table I retrieved values of fit parameters for $6.75 \times 10^{4}$ and $2 \times 10^{6}$ total number of counts from Fig. 2 is given to show the impact on the individual parameters.
TABLE I

Retrieved values of fit parameters for data with different total number of counts $6.75 \times 10^{4}$ and $2 \times 10^{6}$.

\begin{tabular}{c|c|c|c}
\hline \hline Parameter & $\begin{array}{c}\text { Simulated } \\
\text { value }\end{array}$ & $\begin{array}{c}\text { Value for } \\
6.75 \times 10^{4} \text { counts }\end{array}$ & $\begin{array}{c}\text { Value for } \\
2 \times 10^{6} \text { counts }\end{array}$ \\
\hline$\sigma[\mathrm{ns}]$ & 0.1 & 0.102 & 0.100 \\
$t_{0}[\mathrm{~ns}]$ & 6 & 6.00 & 6.00 \\
$\tau_{1}[\mathrm{~ns}]$ & 0.125 & 0.105 & 0.124 \\
$P_{1}[\%]$ & 15 & 14.3 & 14.9 \\
$\tau_{2}[\mathrm{~ns}]$ & 0.5 & 0.489 & 0.499 \\
$P_{2}[\%]$ & 50 & 50.9 & 50.1 \\
$\tau_{3}[\mathrm{~ns}]$ & 5 & 4.90 & 5.00 \\
$P_{3}[\%]$ & 35 & 34.8 & 35.0
\end{tabular}

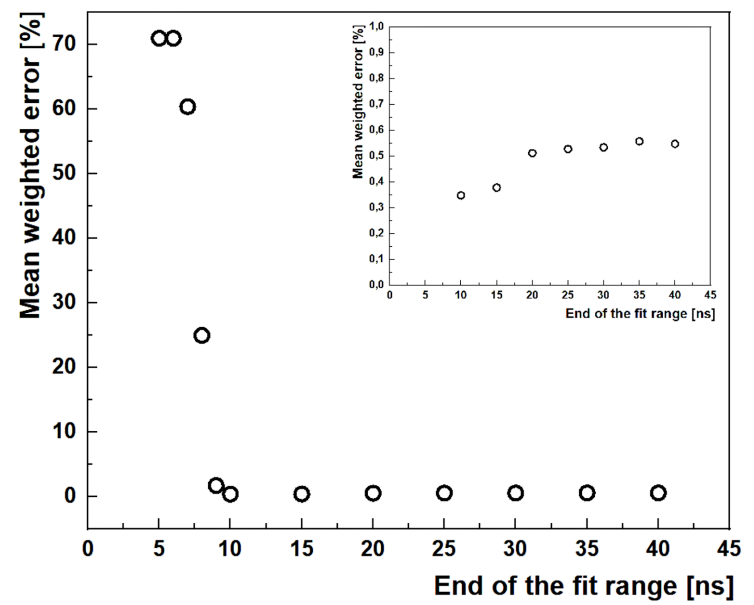

Fig. 4. Influence of the chosen range of the fit to the lifetime spectrum on error made by the fitting procedure.

Figure 3 shows that the lower the width of the bin of lifetime spectrum the smaller error is made by the fitting procedure, which is reasonable, because sensitivity for low lifetime component increases with smaller value of bin width. However, for very small widths sensitivity is deteriorated by increasing number of degrees of the freedom which increases the mean uncertainty of procedure.

The next factor worth checking is the range of the fit. To this end the total number of counts was fixed to $2 \times 10^{6}$ and the bin width was chosen based on Fig. 3 to be $0.02 \mathrm{~ns}$, and initial parameters were equal to simulated ones. Results are shown in Fig. 4. It is worth to remind that the simulated mean of the resolution function was equal to $6 \mathrm{~ns}$ and that it is an estimated position of the maximum of simulated distribution.

In Fig. 4 one can see that very close to the maximum of the distribution error done by the procedure can be of the order of tens of percent. On the other hand, if the range of the fit covers at least one mean lifetime, the fit is very stable and any further changes of the fit range does not significantly influence the results of the fit. 


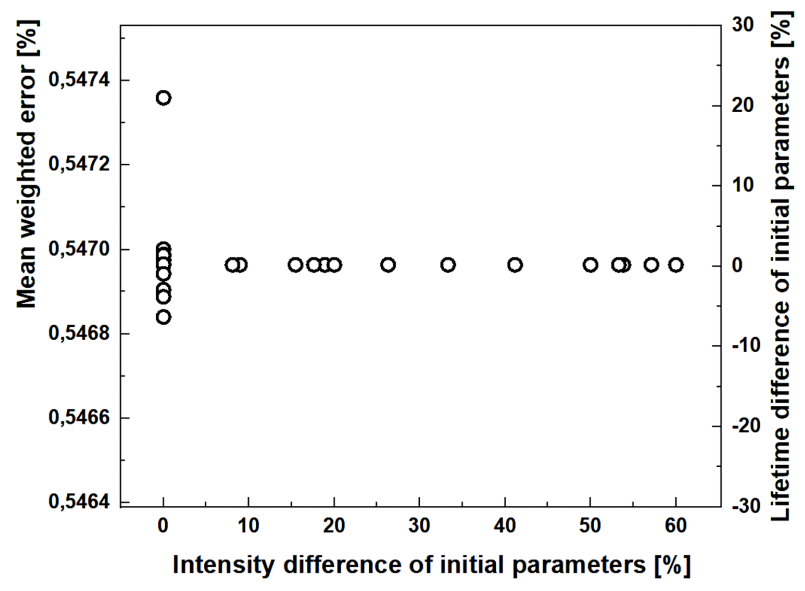

Fig. 5. Influence of difference between initial value of parameters and the simulated ones on error made by fitting procedure.

There is also small decrease of mean error, between 10 and $15 \mathrm{~ns}$, that can point at other effect, not yet considered. The last factor that was checked is the difference of initial values of lifetime and intensity from the simulated ones. Total number of counts were set to 2 million, width of the bin was equal to $0.02 \mathrm{~ns}$ and the fit was done up to 40 ns. Results of the test are shown in Fig. 5.

In Fig. 5 one can see that the mean lifetime has greater impact on the mean uncertainty of the fit than intensity of a given component. However, the changes are small, and results given by the PALS Avalanche software does not depend much on the initial values of fitted parameters.

\section{Conclusions}

PALS Avalanche is a software designed for the J-PET detector to open the possibility to create morphometric images [6, 7]. Simulations of PAL spectra were prepared in order to check the performance of PALS Avalanche. It was checked in view of factors like total number of counts, width of the bin, range of the fit, and initial value of fitted parameters. It was shown that the mean error of a single parameter depends differently for different factors. In proper conditions the mean error made by the fitting procedure of PALS Avalanche for simple positron annihilation systems is of the order of $0.5 \%$. PALS Avalanche software is open for individual tests, and its source is available in [16].

\section{Acknowledgments}

The authors are grateful to Steven D. Bass for correcting the final version of the manuscript. We acknowledge support by the the Foundation for Polish Science through the MPD and TEAM POIR.04.04.00-00-4204/17 programmes, the National Science Centre through the grant No. 2016/21/B/ST2/01222, 2017/25/N/NZ1/00861, the Ministry for Science and Higher Education through grants no. 6673/IA/SP/2016,7150/E-338/SPUB/2017/1, $7150 / \mathrm{E}-338 / \mathrm{M} / 2018$, and $7150 / \mathrm{E}-338 / \mathrm{M} / 2019$.

\section{References}

[1] P. Moskal, Sz. Niedzwiecki, T. Bednarski, et al., Nucl. Instrum. Methods Phys. Res. A A764, 317 (2014).

[2] P. Moskal, N. Zon, T. Bednarski, et al., Nucl. Instrum. Methods Phys. Res. A A775, 54 (2015).

[3] P. Moskal, O. Rundel, D. Alfs, et al., Phys. Med. Biol. 61, 2025 (2016).

[4] Sz. Niedzwiecki, P. Bialas, C. Curceanu, et al. Acta Phys. Pol. B 48, 1567 (2017).

[5] P. Kowalski, W. Wislicki, R.Y. Shopa, et al., Phys. Med. Biol. 63, 165008 (2018).

[6] P. Moskal, D. Kisielewska, C. Curceanu, et al., Phys. Med. Biol. 64, 055017 (2019).

[7] P. Moskal, B. Jasińska, E. Stępień, S.D. Bass, Nature Rev. Phys. 1, 527 (2019).

[8] W. Krzemien, A. Gajos, A. Gruntowski, et al., Acta Phys. Pol. A 127, 1491 (2015).

[9] R. Brun, F. Rademakers, Nucl. Instrum. Methods Phys. Res. A 389, 81 (1997).

[10] BOOST C ++ Libraries.

[11] J. Kansy, Nucl. Instrum. Methods Phys. Res. A 374, 235 (1996).

[12] K. Dulski, B. Zgardzinska, P. Bialas, et al., Acta Phys. Pol. A 132, 1637 (2017).

[13] K. Dulski, C. Curceanu, E. Czerwinski, et al., Hyperfine Interact. 239, 40 (2018).

[14] P. Kirkegaard, M. Eldrup, Comput. Phys. Commun. 3, 240 (1972).

[15] F. James, M. Roos, Comput. Phys. Commun. 10, 343 (1975).

[16] K. Dulski, PALS Avalanche. 